\title{
Establishment and Application of Reverse Transcription Recombinase-Aided Amplification as A Rapid Detection Method for SARS-COV-2
}

\section{Lele Ai}

Centers for Disease Control and Prevention

Wei Liu

chinese PLA center for disease control and prevention

\section{Fuqiang Ye}

Centers for Disease Control and Prevention

\section{Chenxi Ding}

Centers for Disease Control and Prevention

\section{Han Dai}

Eastern General Hospital

\section{Yifang Han}

Centers for Disease Control and Prevention

\section{Changqiang Zhu}

Centers for Disease Control and Prevention

\section{Ruichen LV}

Centers for Disease Control and Prevention

Zhaojiu Han

Centers for Disease Control and Prevention

Xiaobo Zou

Jiangsu University

\section{Yong Qi}

Centers for Disease Control and Prevention

Weilong Tan ( $\square$ njcdc@163.com )

Centers for Disease Control and Prevention

\section{Research article}

Keywords: Recombinase aided amplification, Novel coronavirus, Specificity, Sensitivity

Posted Date: October 23rd, 2020

DOI: https://doi.org/10.21203/rs.3.rs-92818/v1 
License: (c) (i) This work is licensed under a Creative Commons Attribution 4.0 International License. Read Full License 


\section{Abstract}

Background: By the end of August 2020, >23 million cases and 800,000 deaths were attributed to SARSCoV-2 in $>200$ countries. The improvement of simple, rapid, and efficient detection methods is of great significance for the early detection, timely isolation, and protection of susceptible populations. This study aimed to provide an alternative method for the rapid detection of viral nucleic acid.

Methods: This study provided a rapid nucleic acid detection method mediated by recombinant enzyme based on the novel coronavirus (SARS-CoV-2). Primers and probes were designed based on the $\mathrm{Ngene}$ sequence of coronavirus. The method was performed at $39^{\circ} \mathrm{C}$, the detection time was short ( $\left.<20 \mathrm{~min}\right)$, and the detection limit was up to $10^{1}$ copies $/ \mathrm{mL}$.

Results: The primer-probe did not show any cross-reaction with adenovirus, Zika virus, influenza B virus, and chikungunya virus, with good specificity. A total of 106 clinical throat swab samples were compared by reverse transcription recombinase-aided amplification (RT-RAA) and commercial reverse transcriptionquantitative real-time polymerase chain reaction (RT-qPCR); the results were identical.

Conclusions: The novel coronavirus RT-RAA method established in this study had high sensitivity, strong specificity, simple operation, and fast detection speed, and hence, is suitable for the rapid detection of novel coronavirus under the current epidemic situation.

\section{Background}

Coronavirus (CoV) is an enveloped, non-segmented, single-stranded RNA virus belonging to the normal coronavirus subfamily of the family Neviridae [1]. The subfamily consists of $a, \beta, \gamma$, and $\delta$ coronavirus. The viruses are widespread in nature, and their natural hosts include humans and other mammals, such as rats, cats, dogs, and bats [2]. Hitherto, six types of human coronaviruses have been identified [3], including HCOV-229E, HCOV-NL63, HCOV-OC43, HCOV-HKU1, severe acute respiratory syndromeassociated coronavirus (SARS-CoV), and Middle East respiratory syndrome-associated coronavirus (MERS-CoV) [4-5].

SARS-CoV-2 has appeared in Wuhan since December 2019 and caused a new infectious disease named as COVID-19 [6]. Like SARS-CoV and MERS-CoV, SARS-CoV-2 causes fatal pneumonia but is also more capable of person-to-person transmission than SARS-CoV and MERS-CoV [7]. By August 2020, it had infected $>23$ million individuals in more than 200 countries, causing $>800,000$ deaths [8-9]. Therefore, the establishment of a simple, rapid, and reliable detection method for SARS-COV-2 is essential for early diagnosis as well as treatment of patients [10-11] and early adoption of measures to control the epidemic.

At present, quantitative PCR (qPCR) is mainly used to detect viral nucleic acid with high sensitivity and specificity. Since the COVID-19 outbreak [12], many real-time qPCR (RT-qPCR) detection reagents have been developed and used in the laboratory tests for the detection of SARS-CoV-2 [13]. However, RT-qPCR 
requires professional personnel, sophisticated experimental equipment, and a duration of $1.5 \mathrm{~h}$ [14-16]. Currently, the epidemic of SARS-CoV-2 is spreading globally, with a large number of patients, asymptomatic infected individuals, and close contacts. Thus, the rapid and accurate detection of SARSCoV-2 is of vital importance for epidemic prevention and control.

In this study, a single-tube reverse transcription recombinase-aided amplification (RT-RAA) method was improved to detect the SARS-CoV-2 nucleocapsid $(M)$ gene [17]. Due to the normal temperature characteristics of the recombinase, the whole reaction could be carried out at $39{ }^{\circ} \mathrm{C}$ [18]. Moreover, the amplification time could be shortened to $<20$ min due to the absence of traditional PCR heating up and cooling processes. This method can be used to detect SARS-CoV-2 within $30 \mathrm{~min}$, and the detection limit of virus titer is $10^{2}$ RNA copies/reaction, which is similar to that of RT-qPCR. The method showed good specificity in SARS-CoV-2 detection without cross-reactivity with human adenovirus types 3,7 , and 55 and influenza A virus (2009H1N1, H3N2). The commercial RT-qPCR reagent and RT-RAA method were used to detect 106 clinical pharyngeal swab samples, and the results were $100 \%$ consistent. The RT-RAA method established in this study for the detection of SARS-COV-2 is rapid, sensitive, and simple, with low equipment requirement and plays a critical role in the rapid detection of SARS-CoV-2 under the current epidemic situation.

\section{Methods}

\section{RT-RAA primers and probe design}

Various SARS-CoV-2 nucleic acid sequences were downloaded from NCBI database and aligned using DNAMAN 7.0. A conserved region (GenBank: MT559038.1, position 3111-3406) was screened targeting ORF1ab gene [19], which was used to designed RT-RAA primers and probe. Typically, the primer length is $30-35$ bases, the $5^{\prime}$-end should avoid repeated G, the 3 '-end should preferably have $G$ and $C$, and the product length should be $<500$ bases, but $>80$ bases $(100-200$ bases is optimal). In addition, the length of the probe is $46-52$ bases, with at least 30 bases from the $5^{\prime}$-end to tetrahydrofuran and at least 15 bases from the 3 '-end to tetrahydrofuran. The primers and probe were synthesized by Biotechnology (Shanghai) Co., Ltd (Table 1).

\section{Positive templates preparation}

A conserved sequence (GenBank: MT559038.1, position 3111-3406) of the genome of SASR-CoV-2 was selected, and its conservation across strains was evaluated using BLAST on the National Center for Biotechnology Information (NCBI) platform. The sequence was synthesized and cloned into the pET28a vector between restriction endonuclease sites Xba I and Not I by Nanjing GenScript company. The concentration of the recombinant plasmid was quantified using ND-1000 spectrophotometer (Thermo Scientific, Dreieich, Germany), and the DNA copy number was calculated using the following formula: DNA copy number $($ copies $/ \mu \mathrm{L})=\left[6.02 \times 10^{23} \times\right.$ DNA concentration $\left.(\mathrm{g} / \mu \mathrm{L})\right] /[$ [DNA in length $\times 660]$ [20]. The recombinant plasmid was diluted and used as a positive template in the establishment of RT-RAA. The 
recombinant plasmid DNA was digested by restriction endonuclease $\mathrm{Xhol}$, and the linearized DNA was purified using SanPrep Column PCR Product Purification Kit (Sangon Biotech, Shanghai, China). The RNA was transcribed from the purified linearized DNA using an in vitro Transcription T7 Kit (TaKaRa, Dalian, China), purified using a Spin Column RNA Cleanup \& Concentration Kit (Sangon Biotech), and quantified using ND-1000 spectrophotometer (Thermo Scientific). The RNA copy number was calculated using the following formula: RNA copy number $($ copies $/ \mu \mathrm{L})=\left[6.02 \times 10^{23} \times \mathrm{RNA}\right.$ concentration $\left.(\mathrm{g} / \mu \mathrm{L})\right] /[\mathrm{RNA}$ in length×340] [21].

\section{Reaction system of the RT-RAA assay}

The assay was established using the RT-RAA nucleic acid amplification kit (fluorescence) (Jiangsu Qitian Gene Biotechnology Co., Ltd, China). A volume of $42.5 \mu \mathrm{L}$ reaction mixture, consisting of $13 \mu \mathrm{L}$ of $\mathrm{ddH}_{2} \mathrm{O}$, $25 \mu \mathrm{L}$ of buffer $\mathrm{VI}, 2 \mu \mathrm{L}$ of forward primer $(10 \mu \mathrm{M}), 2 \mu \mathrm{L}$ of reverse primer $(10 \mu \mathrm{M})$, and $0.5 \mu \mathrm{L}$ of probe, was added to the fluorescent basic reaction unit, followed by addition of $2.5 \mu \mathrm{L}$ magnesium acetate solution $\left(280 \mathrm{mM}\right.$ ) and $5 \mathrm{~mL}$ template RNA (at a concentration of $10^{4}$ copies $/ \mathrm{mL}$ ). The reaction mixture was transferred to the QT-RAA-F1620 instrument (Jiangsu Qitian Gene Biotechnology), and the amplification conditions were set at $39^{\circ} \mathrm{C}$ for $30 \mathrm{~min}$.

\section{RNA extraction}

Viral nucleic acid was extracted from the pharyngeal swab samples of 106 suspected COVID-19 patients using a viral nucleic acid extraction kit (TaKaRa) according to the instructions of biosafety level II laboratory. The purified nucleic acid was stored at $-80^{\circ} \mathrm{C}$ before use.

\section{Specificity analysis of the SARS-CoV-2 RT-RAA assay}

Nucleic acids of the other five types of respiratory viruses stored in the laboratory were used to verify the specificity of RT-RAA method, including human adenovirus types 3,7 , and 55 , and influenza A virus (2009H1N1 and H3N2) [22]. The nucleic acids of these viruses were extracted under the same nucleic acid extraction condition. Fluorescence RT-PCR detection showed that the Ct values were between 24 and 30 .

\section{Detection limit analysis}

The RNA transcript was diluted and used as a positive template in the evaluation of the detection limit of RT-RAA. Ten-fold serial dilution of the RNA standard, from $10^{6}$ to $10^{0} \mathrm{copies} / \mu \mathrm{L}$, were used as the standards to determine the detection limit of the SARS-CoV-2 RT-RAA assay.

\section{Detecting clinical samples by commercial RT-qPCR and RT-RAA assay}

The pharyngeal swabs were collected from 106 suspected patients of COVID-19, and their nucleic acids were extracted in Biosafety Level 2 laboratory. These samples were detected by the established RT-RAA method and commercial RT-qPCR (ZJ Biotech, Shanghai, China) test kit. 


\section{Results}

\section{Detection limit of the RT-RAA assay}

A total of 7 RNA samples with gradient concentrations from $10^{6}$ to $10^{0}$ copies $/ \mathrm{mL}$ were used as templates to verify the detection limit of the RT-RAA method. The method showed amplification curves with template concentrations $\geq 10^{1} \mathrm{copies} / \mathrm{mL}$, and no amplification curve at $10^{0} \mathrm{copies} / \mathrm{mL}$ (Figure 1). The limit of this detection method was 10 copies/ $\mathrm{mL}$. When the template concentration was $\geq 10^{3}$ copies/ $\mathrm{mL}$, the amplification curve was gained within $10 \mathrm{~min}$.

\section{Specificity analysis of the RT-RAA assay}

The detection method exhibited good specificity, i.e., the results showed that the RT-RAA could detect both the positive control and the positive samples with good amplification curves. However, no amplification curves were detected for adenovirus type 3, type 7, and type 55, influenza A virus (2009H1N1, H3N2), or negative control (Figure 2).

\section{Detection of the clinical Samples}

Nucleic acids extracted from the clinical pharyngeal swab samples of 106 suspected patients were tested using commercial RT-qPCR and RT-RAA detection methods. The results of these two detection methods are consistent (Table 2 and Figure 3 ). Both the sensitivity and specificity of RT-RAA were $100 \%$, as assessed by qPCR, used as the gold standard.

\section{Discussion}

SARS-COV-2, appearing in 2019, is the seventh coronavirus capable of infecting humans and the third fatal coronavirus [6]. It can be transmitted through the respiratory tract, digestive tract, and other routes and causing COVID-19 with a high fatality rate [8]. As of August 2020, more than 23 million individuals in over 200 countries have been infected with SARS-CoV-2, and a total of $>600,000$ deaths because of COVID-19.

SARS-CoV-2 is transmitted mainly by air droplets, thus making it easier for the virus to spread through close contact [23]. When a person is infected with SARS-CoV-2 and shows symptoms, the viral load of the nasopharynx increases in a short duration, which makes the carrier as a virus dissemination center [24]. Early detection, early isolation, and treatment are of great significance to prevent the transmission of the virus. At present, the diagnosis of COVID-19 mainly depends on the RT-qPCR method. Chinese health institutions have developed a large number of RT-qPCR test kits to accurately locate the source of infection and take effective isolation measures. COVID-19 is still spreading rapidly worldwide. However, the method requires sophisticated equipment, professional operators, and long testing time, and hence, many underdeveloped countries cannot carry out large-scale testing. 
RAA/RT-RAA has been widely used in laboratory diagnosis of many pathogenic infections. At present, the existing RAA reagent can detect pathogens, such as Yersinia pestis, Bacillus anthracis, adenovirus, and parvovirus. The RT-RAA method has the characteristics of simple operation, short detection time, and high sensitivity, which is important for the early diagnosis of SARS-CoV-2 infection and implementing hygienic measures [25].

The RT-RAA method of SARS-CoV-2 established in this experiment uses a recombinase-mediated isothermal amplification method to detect SARS-CoV-2 nucleic acids. This method utilizes recombinase to achieve DNA melting and rapid amplification at room temperature, without relying on precision equipment, and the reaction is rapid. The RAA fluorescence detector used in this method is small in size and convenient to carry. An isothermal environment is set at $39^{\circ} \mathrm{C}$. The test can be operated with a lithium battery and is suitable for rapid on-site detection. When the detection of nucleic acid concentration is $>10^{3}$ copies/ $\mu \mathrm{L}$, the detection can be carried out 10 min. The sensitivity of this method is 10 copies/ $\mu \mathrm{L}$, which is close to the detection sensitivity of RT-qPCR. When detecting SARS-CoV-2 positive samples and respiratory viruses using this method, only positive samples showed amplification curves, indicating good specificity of this method. The consistency of the established RT-RAA and commercial RT-qPCR is $100 \%$ in detecting pharyngeal swab samples from 106 suspecting COVID-19 patients, indicating the stability of this method.

The RT-RAA detection method in this study has the advantages of simple operation, short detection time, high sensitivity, and strong specificity as compared to the existing RT-qPCR method, which provides accurate and reliable detection assay for the rapid diagnosis of the COVID-19 epidemic. Thus, for the rapid screening of pathogens in the outbreak, a cost-effective detection method has been proposed [26].

\section{Conclusions}

The novel coronavirus RT-RAA method established in this study was performed at $39^{\circ} \mathrm{C}$, the detection time was short ( $20 \mathrm{~min}$ ), and the detection limit was up to $10^{1} \mathrm{copies} / \mu \mathrm{L}$. The primer-probe did not show any cross-reaction with adenovirus, Zika virus, influenza B virus, and chikungunya virus, with good specificity. The method had high sensitivity, strong specificity, simple operation, and fast detection speed, and hence, is suitable for the rapid detection of novel coronavirus under the current epidemic situation.

\section{Abbreviations}

\section{SARS-CoV-2}

Severe acute respiratory syndrome-associated coronavirus 2; MERS-COV:Middle east respiratory syndrome-associated coronavirus; RT-qPCR:Reverse transcription-quantitative real-time polymerase chain reaction; RT-RAA:Reverse transcription recombinase-aid amplification; COVID-19:Coronavirus disease - 19

\section{Declarations}




\section{Acknowledgements}

No

\section{Authors' contributions}

YQ and W-LT conceived the study. L-LA, WL and F-QY wrote the manuscript and carried out the analysis. C-XD, Y-FH and C-QZ were involved in laboratory works. R-CL and Z-JHcritically revised the manuscript, and all authors approved the submission.

\section{Funding}

This study was supported by Medical Research Topics of the Jiangsu Provincial Health Planning Commission (H2019015) खDevelopment Fund of Key Laboratory of Virology Institute of Wuhan University (2020KF003), Chongqing Research Program of Technology Innovation and Application Development on 2019 Novel Coronavirus-Infected Pneumonia (CSTC2020jscx-fyzx0009).

\section{Availability of data and materials}

All original data and materials are available from the corresponding author upon request.

\section{Ethics approval and consent to participate}

Samples were collected under the approved guidelines and relevant regulations.All experimental procedures were approved by the Ethics Committee of the Research Institute for Medicine, Nanjing Command(Approval number: 2020004) and informed consent were waived. All methods were performed in accordance with the relevant guidelines and regulations.

\section{Consent for publication}

Not applicable.

\section{Competing interests}

The authors declare no competing interests.

\section{Author details}

1. Centre for Diseases Prevention and Control of Eastern Theater, Nanjing, 210002, China

2. Chinese PLA Center for Disease Control and Prevention, Beijing, 100071, China

3. General Hospital of the Eastern Theater Command ,Nanjing, 210002, China

4. Jiangsu university, zhengjiang, 212023, China

\section{References}


1. Cui J, Li F, Shi ZL. Origin and evolution of pathogenic coronaviruses. Nat Rev Microbiol. 2019;17(3):181-92.

2. Zhou P, Yang XL, Wang XG, Hu B, Zhang L, Zhang W, Si HR, Zhu Y, Li B, et al. A pneumonia outbreak associated with a new coronavirus of probable bat origin. Nature. 2020;579:270-3.

3. Du Toit A. Outbreak of a novel coronavirus. Nat Rev Microbiol. 2020;18:123.

4. Hui DS, Azhar EE, Madani TA, Ntoumi F, Kock R, Dar O, Ippolito G, McHugh TD, Memish ZA, Drosten C, et al. The continuing 2019-nCoV epidemic threat of novel coronaviruses to global health-The latest 2019 novel coronavirus outbreak in Wuhan, China. Int J Infect Dis. 2020;91:264-6.

5. Zhu N, Zhang D, Wang W, et al. A novel coronavirus from patients with pneumonia in China,2019. NEnglJ Med. 2020;382:727-33.

6. Wrapp D, Wang N, Corbett KS, Goldsmith JA, Hsieh CL, Abiona O, Graham BS, McLellan JS. Cryo-EM structure of the 2019-nCoV spike in the prefusion conformation. Science. 2020;367:1260-3.

7. Zhu N, Zhang D, Wang W, Li X, Yang B, Song J, Zhao X, Huang B, Shi W, Lu R, et al. A Novel Coronavirus from Patients with Pneumonia in China, 2019. N Engl J Med. 2020;382:727-33.

8. Wu Y. Compensation of ACE2 Function for Possible Clinical Management of 2019-nCoV-Induced Acute Lung Injury. Virol. Sin. 2020. Online ahead of print.

9. Wan Z, Zhang Y, He Z, Liu J, Lan K, Hu Y, Zhang C. A Melting Curve-Based Multiplex RT-qPCR Assay for Simultaneous Detection of Four Human Coronaviruses. Int J Mol Sci. 2016;17:1880.

10. Fan Y, Zhao K, Shi ZL, et al. Bat coronaviruses in China. Viruses. 2019;11(3):210.

11. Menachery VD, Yount BL, Debbink K, et al. A SARS like cluster of circulating bat coronaviruses shows potential for human emergence. Nat Med. 2015;21(12):1508-13.

12. Chu DKW, Pan Y, Cheng SMS, Hui KPY, Krishnan P, Liu Y, Ng DYM, Wan CKC, Yang P, Wang Q, et al. Molecular Diagnosis of a Novel Coronavirus(2019-nCoV) Causing an Outbreak of Pneumonia. Clin Chem. 2020;66:549-55.

13. DeWit E, VanDoremalen N, Falzarano D, et al. SARS and MERS: recent insights into emerging coronaviruses. Nat Rev Microbiol. 2016;14(8):523-34.

14. Li Y, Wan Z, Hu Y, Zhou Y, Chen Q, Zhang C. A mismatch-tolerant RT-quantitative PCR: Application to broad-spectrum detection of respiratory syncytial virus. Biotechniques. 2019;66:225-30.

15. Wu F, Zhao S, Yu B, Chen YM, Wang W, Song ZG, Hu Y, Tao ZW, Tian JH, Pei YY, et al. A new coronavirus associated with human respiratory disease in China. Nature. 2020;579:265-9.

16. Huang C, Wang Y, Li X, et al. Clinical features of patients infected with 2019 novel coronavirus in Wuhan, China. The Lancet. 2020;395(10223):497-506.

17. Ceraolo C, Giorgi FM. Genomic variance of the 2019-nCoV coronavirus. J Med Virol. 2020;92:522-8.

18. Lu R, Zhao X, Li J, et al. Genomic characterisation and epidemiology of 2019 novel coronavirus: implications for virus origins and receptor binding. The Lancet. 2020;395:565-74.

19. Li Y, Zhou Y, Ma Y, Xu R, Jin X, Zhang C. A Mismatch-tolerant RT-LAMP Method for Molecular Diagnosis of Highly Variable Viruses. Bio-Protocol. 2019;9:e3415. 
20. Riou J, Althaus CL. Pattern of early human-to-human transmission of Wuhan 2019 novel coronavirus (2019-nCoV), December 2019 to January 2020. Eurosurveillance 2020, 25, 2000058.

21. Forootan A, Sjöback R, Bjorkman J, Sjögreen B, Linz L, Kubista M. Methods to determine limit of detection and limit of quantification in quantitative real-time PCR (qPCR). Biomol Detect Quantif. 2017;12:1-6.

22. Kuypers J, Jerome KR. Applications of digital PCR for clinical microbiology. J Clin Microbiol. 2017;55:1621-8.

23. Wang $\mathrm{H}$, Cong $\mathrm{F}$, Zeng $\mathrm{F}$, et al. Development of a real time reverse transcription loop-mediated isothermal amplification method (RT-LAMP) for detection of a novel swine acute diarrhea syndrome coronavirus (SADS-CoV) [Research Support, Non-U.S. Gov't]. J Virol Methods. 2018 Oct;260:45-8.

24. Ahn SJ, Baek YH, Lloren KKS, et al. Rapid and simple colorimetric detection of multiple influenza viruses infecting humans using a reverse transcriptional loopmediated isothermal amplification (RTLAMP) diagnostic platform. BMC Infect Dis.2019Aug1;19(1):676.

25. Liu X, Yan Q, Huang J, Chen J, Guo Z, Liu Z, et al. Influence of design probe and sequence mismatches on the efficiency of fluorescent RPA. World J Microbiol Biotechnol. 2019;35(6):1-11.

26. Corman VM, Landt O, Kaiser M, Molenkamp R, Meijer A, Chu DKw, Bleicker T, Brünink S, Schneider J, Schmidt ML, Mulders DGjc, Haagmans BL, vanderVeer B, VandenBrink S, Wijsman L, Goderski G, Romette JL, Ellis J, Zambon M, Peiris M, Goossens H, Reusken C. Koopmans MPg, Drosten C. Detection of 2019 novel coronavirus (2019-nCoV) by real-time RT-PCR[J]. Eurosurvllance, 2020, 25(3).

\section{Tables}

Table 1

RT-RAA primers and probe for the detection of SARS-CoV-2

\begin{tabular}{|llll|}
\hline $\begin{array}{l}\text { Target } \\
\text { gene }\end{array}$ & $\begin{array}{l}\text { Primer } \\
\text { name }\end{array}$ & Sequence $\left(\mathbf{5}^{\prime} \mathbf{3}^{\prime} \mathbf{3}^{\prime}\right)$ & $\begin{array}{c}\text { Length } \\
\text { (bp) }\end{array}$ \\
\hline ORF1ab & NCoV-F & $5^{\prime}$-GCGAACAATTCAACAGACACTGTAGACACAGTAC-3' & 34 \\
\cline { 2 - 4 } & $\begin{array}{l}\text { NCoV- } \\
\mathrm{R}\end{array}$ & $5^{\prime}$-CTAGGTGTTTCCACAATGTAGGACCATGAGC-3' & 31 \\
\cline { 2 - 4 } & $\begin{array}{l}\text { NCoV- } \\
\text { pro }\end{array}$ & $\begin{array}{l}5^{\prime} \text {-CAACCTCAATTAGAGATGGAACTTACACCAGT(FAM-dT) (THF) } \\
(\text { BHQ-dT) TCAGACTATTGAAGTG-3' }\end{array}$ & 51 \\
\hline
\end{tabular}


Table 2

Comparison of the RT-RAA assay with a commercial RT-qPCR assay

\begin{tabular}{|c|c|c|c|c|c|c|}
\hline & & \multicolumn{2}{|c|}{ RT-RAA assay } & \multirow[t]{2}{*}{ Total } & \multirow{2}{*}{$\begin{array}{l}\text { Positive rate } \\
\text { (\%) }\end{array}$} & \multirow{2}{*}{$\begin{array}{l}\text { Concordance rate } \\
(\%)\end{array}$} \\
\hline & & Positive & Negative & & & \\
\hline \multirow{4}{*}{$\begin{array}{l}\text { RT-qPCR } \\
\text { assay }\end{array}$} & Positive & 32 & 0 & 32 & \multirow[t]{2}{*}{$30.1 \%$} & \multirow[t]{2}{*}{$100 \%$} \\
\hline & Negative & 0 & 74 & 74 & & \\
\hline & Total & 32 & 74 & & & \\
\hline & $\begin{array}{l}\text { Positive rate } \\
(\%)\end{array}$ & $30.1 \%$ & & & & \\
\hline
\end{tabular}

Figures

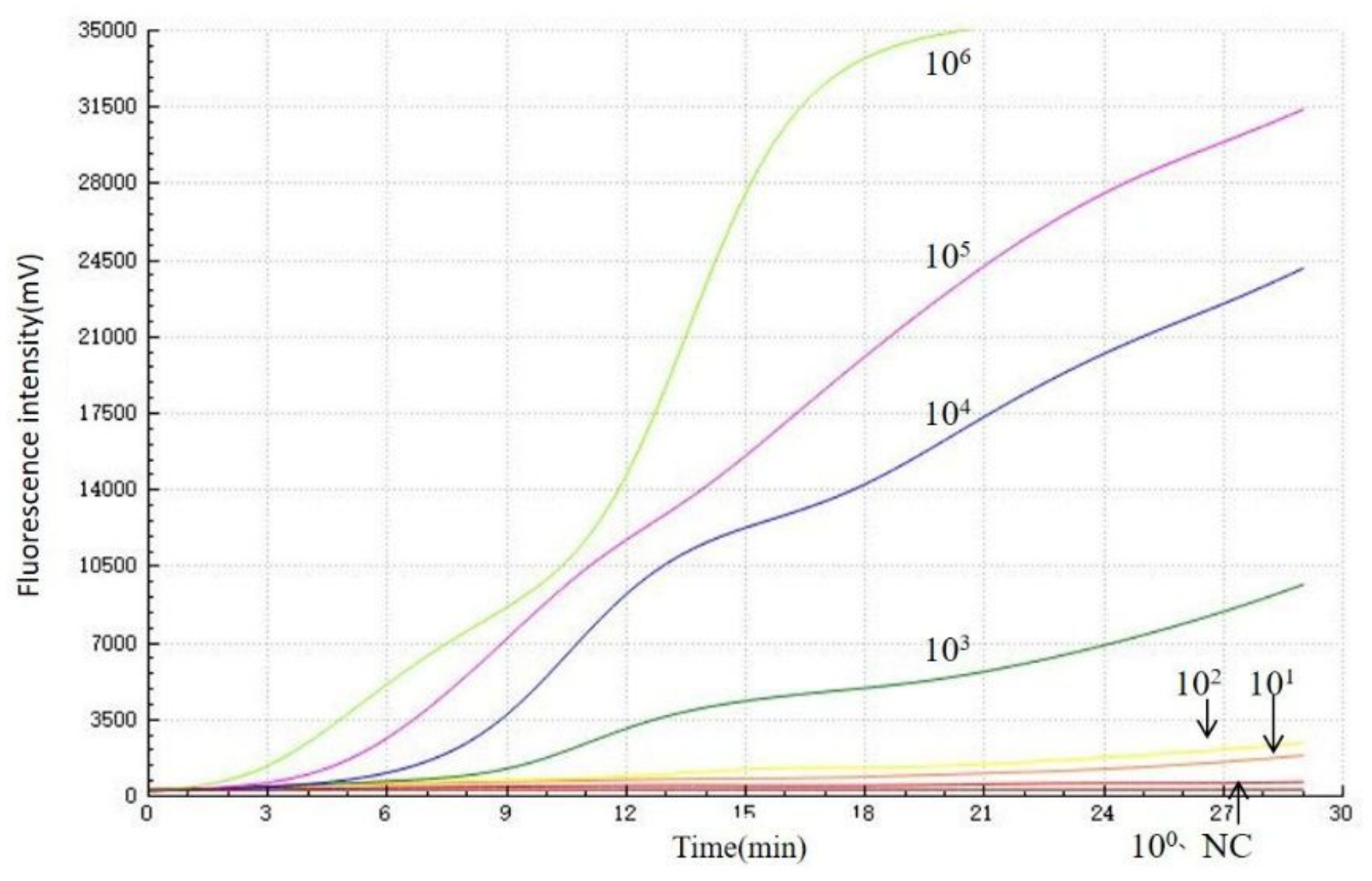

\section{Figure 1}

Detection limit analysis of the RT-RAA assay. The template concentration ranged from 106 to 100 copies/ $\mu \mathrm{L}$. NC, negative control. 


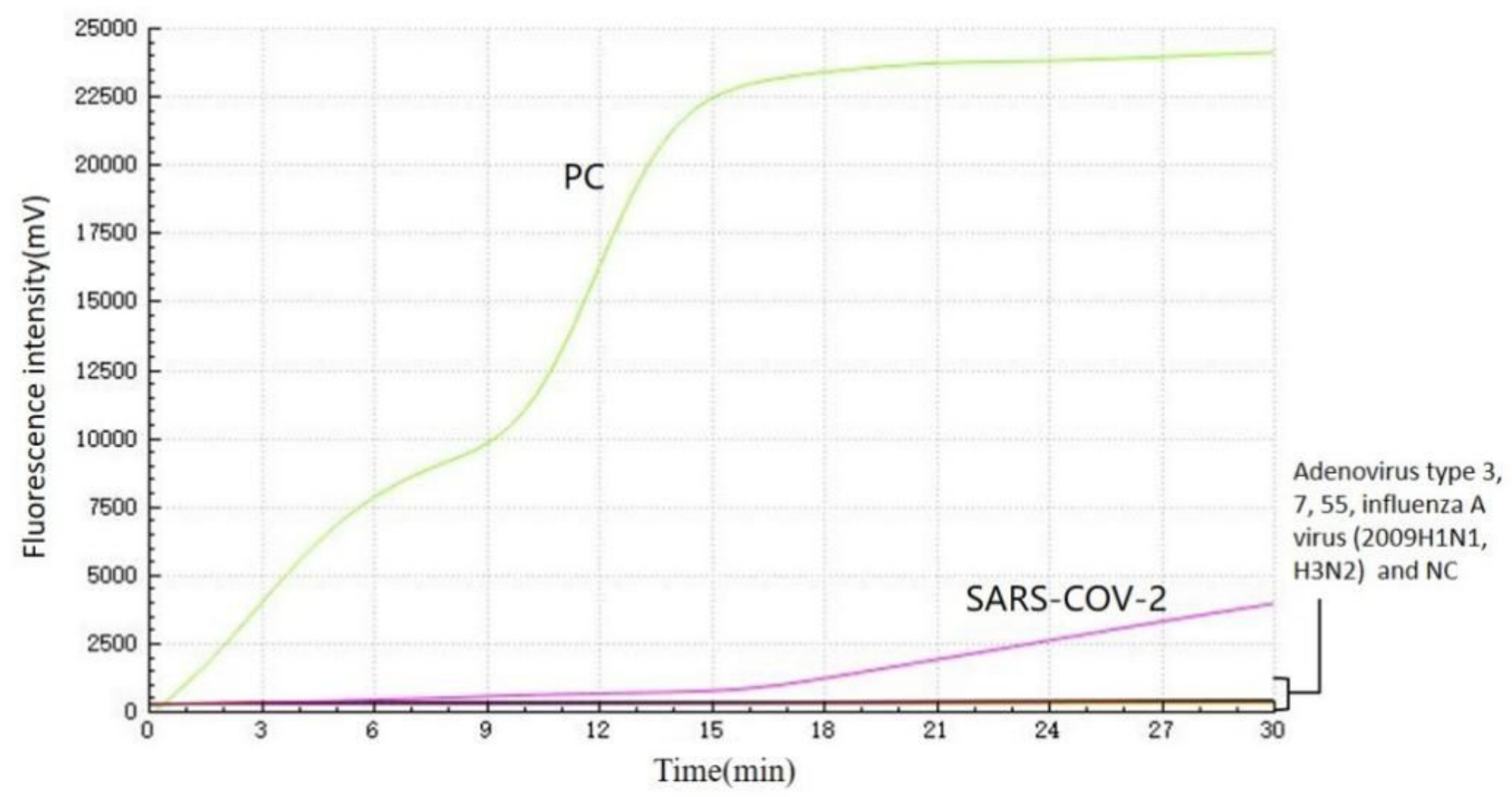

Figure 2

Specificity analysis of the RT-RAA assay. The common respiratory viruses used in this assay included adenovirus type $3,7,55$; influenza A virus (2009H1N1, H3N2). PC represents positive control. NC represents negative control.
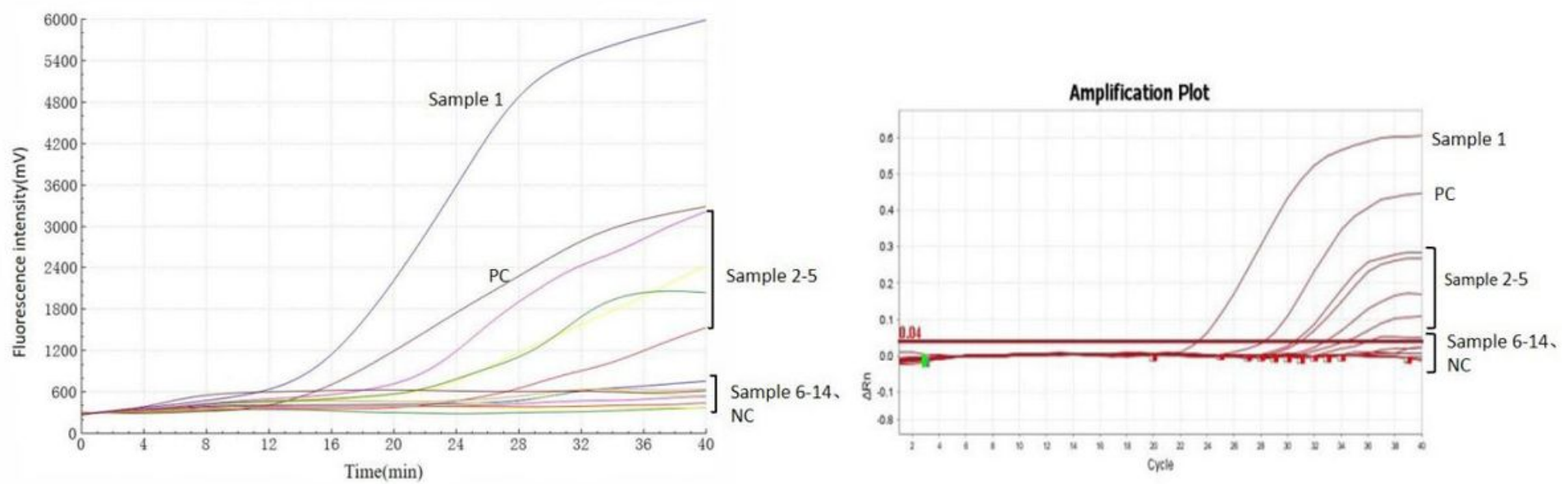

A

Figure 3 
Clinical samples were tested by RT-RAA (A) and commercial RT-QPCR assay (B). Samples 1-5 are positive, samples 6-14 are negative, and the results of the two methods are completely consistent. PC represents positive control. NC represents negative control. 